\title{
ELASTIC PLASTIC ANALYSIS OF SHALLOW SHELLS-A NEW APPROACH
}

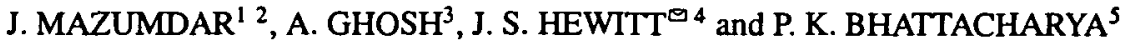

(Received 11 February, 2001; revised 6 May, 2005)

\begin{abstract}
A simple and efficient method for the analysis of the elastic-plastic bending of shallow shells is presented. The method is based upon the concept of contour lines of equal deflection on the surface of the shell, and uses Illyushin's theory of plastic deformation. As an illustration of the method, a technically interesting example of a shallow elliptic elastic dome is examined. Results are obtained for increasing loads and varying aspect ratios, and are illustrated graphically. The application of the method to other shell geometries is quite straightforward.
\end{abstract}

\section{Introduction}

The widespread use of shallow shell structures in engineering and building design has naturally given rise to a need for reliance upon a method of analysis of shallow shells which can adequately account for arbitrary geometric plan form. Analytic techniques have serious limitations because of the difficulties involved in solving nonlinear differential equations. Therefore, despite the simplified nature of shallow shell theory, relatively few solutions are known $[1,2,3,7]$.

The present work may be considered as a sequel to earlier work $[6,9]$ where a new approach for the elastic-plastic analysis of plates of arbitrary shape has been proposed. The present paper, like the previous ones, uses the concept of contour lines of equal

\footnotetext{
${ }^{1}$ School of Mathematical Sciences, The University of Adelaide, Adelaide SA 5005, Australia; e-mail: jmazumdar@maths.adelaide.au.

${ }^{2}$ School of Electrical and Information Engineering, University of South Australia, Mawson Lakes Boulevard, Mawson Lakes SA 5095, Australia;e-mail: Jagannath.Mazumdar@unisa.edu.au.

${ }^{3}$ School of Mathematical Sciences, The University of Adelaide, Adelaide SA 5005, Australia; e-mail: aghosh@maths.adelaide.au.

${ }^{4}$ School of Mathematics and Statistics, University of South Australia, Mawson Lakes Boulevard, Mawson Lakes SA 5095, Australia; e-mail: John.Hewitt@unisa.edu.au.

${ }^{5}$ Department of Mathematics, Indian Institute of Technology, Delhi-110016, India.

(C) Australian Mathematical Society 2005, Serial-fee code 1446-1811/05
} 
deflection, that is, contour lines which are obtained by intersecting the bent surface by a set of horizontal planes parallel to the base of the shell. This same concept is used in the present investigation in order to analyse the elastic-plastic bending of shallow shells using Illyushin's theory of plastic deformation [5]. The advantage in using contour lines of equal deflection is that the partial differential equations involved in shell analysis can be transformed into ordinary differential equations. These are more amenable to numerical solution where nonlinearity prevents an analytic solution.

In the following work, the force and moment equations of Vlasov shallow shell theory [11] are transformed into ODE's involving the shell vertical deflection and stress function. The method is then illustrated by considering the elastic-plastic bending of a shallow dome on an elliptical base with constant load. Central deflection response with load for different aspect ratios is examined. Also, the variation of the plastic strain at the centre and edge of the shell are studied for different aspect ratios.

\section{Theory and derivation of basic equations}

Consider a shallow shell of uniform thickness $h$ subject to a continuously distributed normal load. Let the equation of the middle surface of the shell, referred to a system of orthogonal coordinates $(x, y, z)$, be given by

$$
z=\frac{x^{2}}{2 R_{x}}+\frac{x y}{R_{x y}}+\frac{y^{2}}{2 R_{y}},
$$

where the shell will be called shallow if $r=\sqrt{x^{2}+y^{2}}$ is small compared to the least $R_{x}, R_{y}$ and $R_{x y}$ (the radii of curvature) everywhere in the region, and if $R_{x}, R_{y}$ and $R_{x y}$ can be taken as constants.

When the shell is acted upon by a transverse load $q(x, y)$ then the intersections between the deflected surface and the parallels $z=$ constant yield contours which, after projection onto the base plane, are a set of level curves $u(x, y)=$ constant, which are isodeflection contour lines. If the boundary of the shell is subjected to a combination of clamping and simple support so that it does not move in a direction perpendicular to the base plane, then clearly the boundary, assumed to be a simple closed curve $c$, will belong to the family of lines of equal deflection and without loss of generality, one may consider that $u=0$ on the boundary.

Consider the equilibrium of an element of the shell bounded by any contour lines of equal deflection. The conditions for the equilibrium of an element of the shell require that the sum of moments about the tangent line at any point to the curve $u(x, y)=$ constant of all the forces acting on the element and the sum of all the forces normal to the plane $z=0$ to vanish. Therefore proceeding in the same way as in [7], 
we obtain

$$
\begin{aligned}
\sum M= & \mathbf{n}_{0} \cdot \oint M_{n} \mathbf{n} d s+\mathbf{n}_{0} \cdot \oint V_{n} \mathbf{r}_{0} d s \\
& -\mathbf{n}_{0} \cdot \iint\left[q-\frac{N_{x}}{R_{x}}-\frac{N_{y}}{R_{y}}-\frac{2 N_{x y}}{R_{x y}}\right] \mathbf{r} d x d y=0
\end{aligned}
$$

and

$$
\sum Z=\oint V_{n} d s-\iint\left[q-\frac{N_{x}}{R_{x}}-\frac{N_{y}}{R_{y}}-\frac{2 N_{x y}}{R_{x y}}\right] d x d y=0,
$$

where $\left(x_{0}, y_{0}\right)$ is a fixed point on the line $u=$ constant, $\mathbf{n}$ and $\mathbf{n}_{0}$ denote the unit vectors normal to this line at any arbitrary point $(x, y)$ and at the fixed point $\left(x_{0}, y_{0}\right)$ respectively, and $\mathbf{r}$ and $\mathbf{r}_{0}$ denote the position vectors from the fixed point $\left(x_{0}, y_{0}\right)$ to any arbitrary point inside the contour and on the contours respectively.

We use Illyushin's theory of elastic-plastic deformation [5], which is for small deformations and easy in application. Russian scientists based their work on this theory on a deformation theory of plasticity, rather than flow theory. Use of a deformation law predicts results close to those of a flow law, and can provide useful indications of obtaining solutions by flow theories [4]. The theory defines a function $\Omega$ as

$$
\Omega= \begin{cases}0, & \text { when } e \leq 1 \text { and the region is elastic, } \\ \lambda\left(1-3 /(2 e)+1 /\left(2 e^{3}\right)\right), & \text { when } e \geq 1 \text { and the region is plastic. }\end{cases}
$$

Here

$$
e=\frac{h}{\sqrt{3 e_{s}}}\left(w_{x x}^{2}+w_{y y}^{2}+w_{x x} w_{y y}+w_{x y}^{2}\right)^{1 / 2}
$$

in which $e_{s}$ is the yield strain and $\lambda$ is a material constant.

The well-known expressions for the bending moments $M_{x}, M_{y}$ and $M_{x y}$ and the shearing forces $Q_{x}$ and $Q_{y}$, see Timoshenko and Woinowsky-Krieger [10], are then multiplied by $(1-\Omega)$, so that they are modified only in the plastic case when $e \geq 1$.

The membrane forces $N_{x}, N_{y}$ and $N_{x y}$ are determined by

$$
N_{x}=\frac{\partial^{2} \phi}{\partial y^{2}}, \quad N_{y}=\frac{\partial^{2} \phi}{\partial x^{2}} \quad \text { and } \quad N_{x y}=\frac{\partial^{2} \phi}{\partial x \partial y},
$$

where $\phi$ is the stress function. Making use of these relations, and proceeding as in Mazumdar [8], (2.2) finally reduces to

$$
\begin{aligned}
& \frac{d^{3} w}{d u^{3}} \oint(1-\Omega) R d s+\frac{d^{2} w}{d u^{2}} \oint(1-\Omega) F d s+\frac{d w}{d u} \oint(1-\Omega) G d s \\
& \quad+\frac{d^{2} w}{d u^{2}} \oint D\left(\Omega_{x} u_{x}-\Omega_{y} u_{y}\right) \sqrt{t} d s+\frac{d w}{d u} \oint \frac{D}{\sqrt{t}}\left(\Omega_{x} K+\Omega_{y} L\right) d s
\end{aligned}
$$




$$
-\iint\left[q-\frac{1}{R_{x}} \frac{\partial^{2} \phi}{\partial y^{2}}-\frac{1}{R_{y}} \frac{\partial^{2} \phi}{\partial x^{2}}+\frac{2}{R_{x y}} \frac{\partial^{2} \phi}{\partial x \partial y}\right] d x d y=0,
$$

where the expressions for $t, R, F, G, K, L$ and $H$ are given in [6].

It is to be noted that the moment equation (2.1) and the force equation (2.2) are identical when $u(x, y)=$ constant is the correct form of lines of equal deflection.

The condition for the continuity of deformation, a reduced form of the BeltramiMichell compatibility condition, in this case reduces to

$$
\nabla^{4} \phi=\frac{12 D\left(1-v^{2}\right)}{h^{2}}(1-\Omega)\left(\frac{1}{R_{x}} \frac{\partial^{2} w}{\partial y^{2}}+\frac{1}{R_{y}} \frac{\partial^{2} w}{\partial x^{2}}-\frac{2}{R_{x y}} \frac{\partial^{2} w}{\partial x \partial y}\right) .
$$

Thus our problem now reduces to solving (2.3) and (2.4) for $w$ and $\phi$ giving the true equations for the lines of constant deflection.

In the above equations $\Omega$ is a function of $e$, which in turn is a function of $u$. Hence (2.4) can be transformed into an integro-differential equation by using Green's theorem, as has been shown in the case of elastic shells [7].

Since the intrinsic geometry of the middle surface of the shell is considered to be Euclidean, we will assume that the deflection contours are as for the corresponding flat plate problems.

Further, since (2.4) must hold for all points in the interior of the shell, by integrating we get

$$
\iint\left[\nabla^{4} \phi-\frac{12 D\left(1-v^{2}\right)}{h^{2}}(1-\Omega)\left(\frac{1}{R_{x}} \frac{\partial^{2} w}{\partial y^{2}}+\frac{1}{R_{y}} \frac{\partial^{2} w}{\partial x^{2}}-\frac{2}{R_{x y}} \frac{\partial^{2} w}{\partial x \partial y}\right)\right] d x d y=0,
$$

which after applying Green's theorem reduces to

$$
\begin{aligned}
& \oint \nabla\left(\nabla^{2} \phi\right) \cdot \mathbf{n} d s \\
& \quad-\frac{12 D\left(1-v^{2}\right)}{h^{2}}(1-\Omega) \oint\left(K_{x} \frac{\partial w}{\partial y} \frac{u_{y}}{\sqrt{t}}+K_{y} \frac{\partial w}{\partial x} \frac{u_{x}}{\sqrt{t}}\right) d s=0 .
\end{aligned}
$$

Assuming as before that $\phi$ is a function of $u,(2.5)$ finally reduces to

$$
\begin{aligned}
\frac{d^{3} \phi}{d u^{3}} \oint R d s+\frac{d^{2} \phi}{d u^{2}} \oint F d s+\frac{d \phi}{d u} \oint G d s & \\
& -\frac{12 D\left(1-v^{2}\right)}{h^{2}}(1-\Omega) \frac{d w}{d u} \oint \frac{K_{x} u_{y}^{2}+K_{y} u_{x}^{2}}{\sqrt{t}} d s=0
\end{aligned}
$$

where $K_{x}$ and $K_{y}$ denote curvatures at a point, and $K_{x y}$ is taken to be zero in accordance with the shallow shell theory.

Equations (2.3) and (2.6) are now two basic equations for our shallow shell analysis. In the next section we will use these equations to illustrate a few important examples. 


\section{Illustration}

As an illustration consider here the bending of (i) a clamped and (ii) a simply supported shallow dome of non-zero Gaussian curvature upon an elliptic base. The geometry of the shell is as described in Figure 1. The first approximation for the lines of constant deflection for this case may be reasonably taken as

$$
u=1-\frac{x^{2}}{a^{2}}-\frac{y^{2}}{b^{2}}=\text { constant }
$$

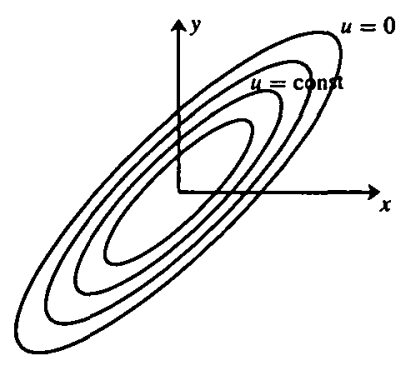

(a) Contour lines of equal deflection.

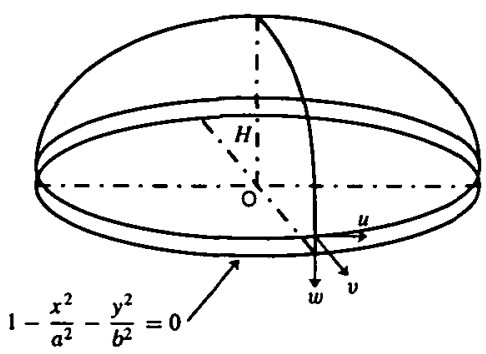

(b) Shallow dome upon an elliptic base.

FIGURE 1.

Performing our contour integrations taken around the closed contour and the double integration over the ellipse

$$
\frac{x^{2}}{a^{2}}+\frac{y^{2}}{b^{2}}=1-u
$$

(2.3) now reduces to

$$
\begin{aligned}
& (1-\Omega)(1-u) \frac{d^{3} w}{d u^{3}}-2(1-\Omega) \frac{d^{2} w}{d u^{2}} \\
& -\frac{d \Omega}{d u}\left[(1-u) \frac{d^{2} w}{d u^{2}}-2 P\left(\frac{1}{a^{4}}+\frac{1}{b^{4}}+\frac{2 v}{a^{2} b^{2}}\right) \frac{d w}{d u}\right]+q_{1}-\frac{\gamma}{D} \frac{d \phi}{d u}=0,
\end{aligned}
$$

where $P=a^{4} b^{4} /\left(3 a^{4}+2 a^{2} b^{2}+3 b^{4}\right)$ and $q_{1}=q P / 2 D$. Equation (2.6) will reduce to

$$
(1-u) \frac{d^{3} \phi}{d u^{3}}-2 \frac{d^{2} \phi}{d u^{2}}+E h(1-\Omega) \gamma \frac{d w}{d u}=0
$$

with $\gamma=P\left(K_{x} / b^{2}+K_{y} / a^{2}\right)$. 
3.1. The boundary conditions The satisfaction of gross boundary conditions, that is, the conditions on resultant forces or moments of the displacements of one surface such as the middle surface, should be sufficient for problems involving what have been defined as thin shells, but if it seems necessary to satisfy more complete conditions at every point of cross sections, many of the supplementary methods and solutions used for thin plates can be applied with reasonable accuracy to most shell problems [3].

For the present illustrations, the boundary conditions imposed on $w$ and $\phi$ are

$$
\left.w \cdot\right|_{u=0}=0=\left.\frac{d w}{d u}\right|_{u=0}
$$

and

$$
\left.\phi\right|_{u=0}=0=\left.\frac{d \phi}{d u}\right|_{u=0}
$$

for clamped edge conditions.

For a simply supported edge, $\left.w\right|_{u=0}=0=\left.M_{n}\right|_{u=0}$ with similar conditions for the stress function $\phi$.

3.2. Method of solution Using the non-dimensional parameters

$$
w^{*}=\frac{w h}{e_{s} a^{2}}, \quad Q^{*}=\frac{q a^{2} h}{2 D e_{s}}, \quad \phi^{*}=\frac{\phi}{E e_{s} a^{2} h} \quad \text { and } \quad E h=\frac{12\left(1-v^{2}\right) D}{h^{2}},
$$

the nonlinear differential equation (3.2) and Equation (3.3) are transformed into

$$
\begin{gathered}
(1-\Omega)\left[(1-u) \frac{d^{3} w^{*}}{d u^{3}}-2 \frac{d^{2} w^{*}}{d u^{2}}\right]-\frac{d \Omega}{d u}\left[(1-u) \frac{d^{2} w^{*}}{d u^{2}}-2 P_{1} \frac{d w^{*}}{d u}\right] \\
-12\left(1-v^{2}\right) \frac{\gamma}{h} \frac{d \phi^{*}}{d u}=-Q^{*} \frac{P}{a^{4}}, \\
P_{1}=P\left(1 / a^{4}+1 / b^{4}+2 v / a^{2} b^{2}\right) \text { and } \\
(1-u) \frac{d^{3} \phi^{*}}{d u^{3}}-2 \frac{d^{2} \phi^{*}}{d u^{2}}+(1-\Omega) \frac{\gamma}{h} \frac{d w^{*}}{d u}=0 .
\end{gathered}
$$

On substitution of the value of $\Omega,(3.5)$ and (3.6) will now reduce to

$$
\begin{aligned}
\{(1 & \left.-u) \frac{d^{3} w^{*}}{d u^{3}}-2 \frac{d^{2} w^{*}}{d u^{2}}\right\} \frac{2 e^{3}(1-\lambda)+\lambda\left(3 e^{2}-1\right)}{2 e^{3}} \\
& -\frac{\lambda}{4} \frac{\left(e^{2}-1\right)}{e^{5}} a^{4}\left[2 M \frac{d w^{*}}{d u} \frac{d^{2} w^{*}}{d u^{2}}+N\left(\frac{d^{2} w^{*}}{d u^{2}}\right)^{2}+N \frac{d w^{*}}{d u} \frac{d^{3} w^{*}}{d u^{3}}\right. \\
& \left.+2 t^{2} \frac{d^{2} w^{*}}{d u^{2}} \frac{d^{3} w^{*}}{d u^{3}}\right]\left[(1-u) \frac{d^{2} w^{*}}{d u^{2}}-2 P_{1} \frac{d w^{*}}{d u}\right]
\end{aligned}
$$




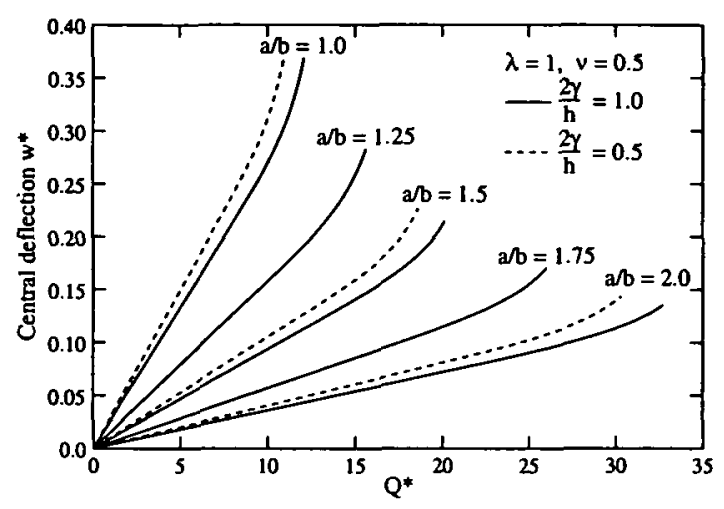

FIGURE 2. Variation of central deflection $w^{*}$ with $Q^{*}$ for varying $\gamma / h$.

$$
-12\left(1-v^{2}\right) \frac{\gamma}{h} \frac{d \phi^{*}}{d u}-\frac{Q^{*} P}{a^{4}}=0
$$

and

$$
(1-u) \frac{d^{3} \phi^{*}}{d u^{3}}-2 \frac{d^{2} \phi^{*}}{d u^{2}}+\frac{2 e^{3}(1-\lambda)+\lambda\left(3 e^{2}-1\right)}{2 e^{3}} \frac{\gamma}{h} \frac{d w^{*}}{d u}=0
$$

where $e^{2}$ becomes

$$
\begin{aligned}
e^{2}= & \frac{1}{3}\left[\left\{4 c_{1}\left(\frac{d w^{*}}{d u}\right)^{2}-8 c_{1}(1-u) \frac{d w^{*}}{d u} \frac{d^{2} w^{*}}{d u^{2}}+4 c_{3}(1-u)^{2}\left(\frac{d^{2} w^{*}}{d u^{2}}\right)^{2}\right\}\right. \\
& -8\left\{(1-u) c_{2} \frac{d w^{*}}{d u} \frac{d^{2} w^{*}}{d u^{2}}-c_{2}(1-u)^{2}\left(\frac{d^{2} w^{*}}{d u^{2}}\right)^{2}\right\} \cos 2 \alpha \\
& \left.+4\left\{c_{4}(1-u)^{2}\left(\frac{d^{2} w^{*}}{d u^{2}}\right)^{2}\right\} \cos ^{2} 2 \alpha\right],
\end{aligned}
$$

$\alpha$ denoting the eccentric angle at any point on the contour of the ellipse given by (3.1), and

$$
\begin{array}{ll}
c_{1}=\left(1+\beta^{2}+\beta^{4}\right), & c_{2}=\left(1-\beta^{4}\right), \\
c_{3}=\left(1+\beta^{2}\right)^{2}, & c_{4}=\left(1-\beta^{2}\right)^{2} \text { and } \beta=a / b .
\end{array}
$$

Equations (3.7) and (3.8) are to be solved for $w^{*}$ and $\phi^{*}$. However, they do not appear to yield exact solutions and hence one has to adopt some procedure for approximate solution.

The method used for solution was a Runge-Kutta iterative scheme using the boundary conditions of (3.4), combined with a shooting method in order to satisfy the 


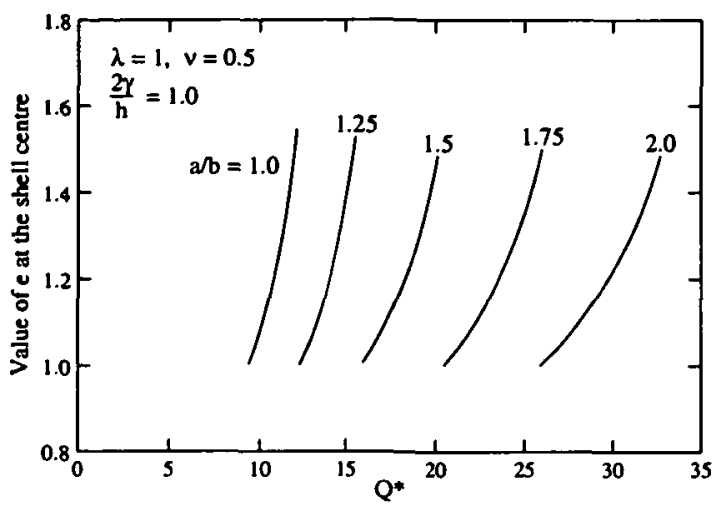

FIGURE 3. Variation of $e$ at the centre with $Q^{*}$ for varying aspect ratio.

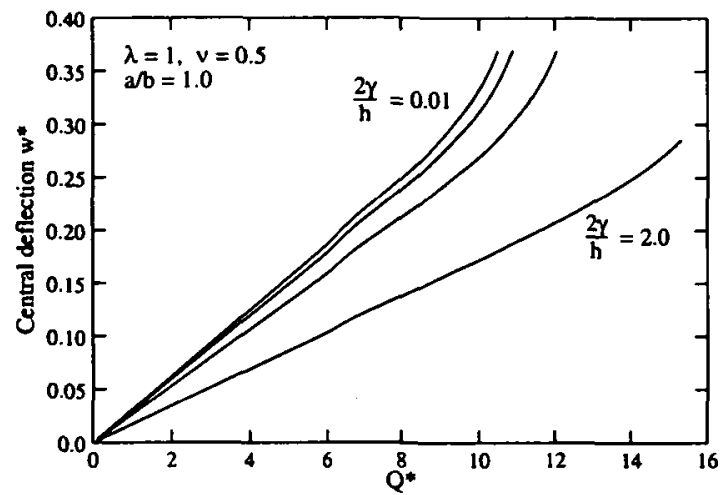

FIGURE 4. Variation of central deflection $w^{*}$ with $Q^{*}$ for varying $\gamma / h$.

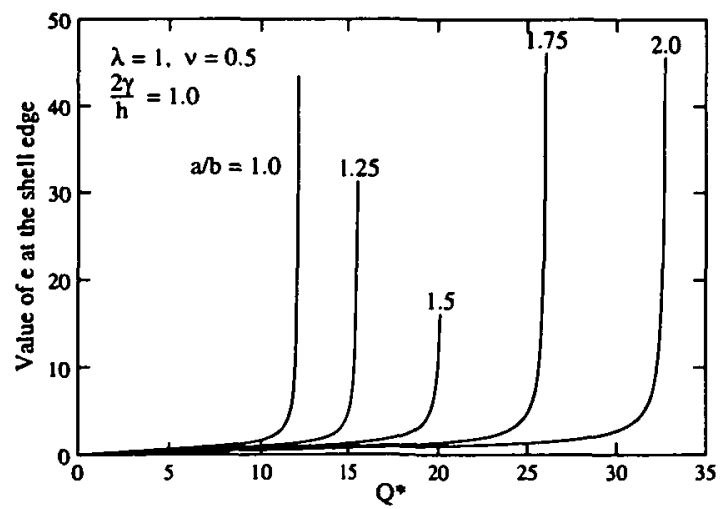

Figure 5. Variation of $e$ at the shell edge with $Q^{*}$. 
remaining boundary conditions

$$
\left.\sqrt{1-u} \frac{d w^{*}}{d u}\right|_{u=1}=0 \quad \text { and }\left.\quad \sqrt{1-u} \frac{d \phi^{*}}{d u}\right|_{u=1}=0 .
$$

Figure 2 shows the deflection $w^{*}$ at the centre of the shell for aspect ratios $a / b=$ $1.0,1.25,1.5,1.75$ and 2.0 for $2 \gamma / h=1.0$, and $a / b=1.0,1.5$ and 2.0 for $2 \gamma / h=$ 0.5 . The central deflection decreases as $a / b$ increases and also as $2 \gamma / h$ increases.

Figure 3 shows the variation in plastic strain $e$ at the centre of the shell versus the load $Q^{*}$. At the maximum value of $e$ in each case, the shell has reached the limit of elastic-plastic response at the edge $u=0$, where $e$ is much greater, as seen in Figure 5 .

The case $2 \gamma / h \rightarrow 0$ gives the response for a plate. Figure 4 shows that there is little change in $w^{*}$ for $2 \gamma / h$ varying from 0 to 0.5 , but increasing as $2 \gamma / h$ increases to 2. At the maximum value of $w^{*}$ for $2 \gamma / h=2.0$ the response at the centre of the shell is still elastic, while the limit of elastic plastic response at the edge $u=0$ has been reached.

\section{Conclusions}

A new and uncomplicated method for the elastic plastic analysis of shallow shells is presented. The method combines the use of contour lines of equal deflection with Illyushin's method of determining small plastic deformation. The method is illustrated using the example of the bending of an elliptical dome. The procedure presented can easily be adopted for other geometries.

\section{Acknowledgements}

The authors are grateful to Dr M. M. Banerjee for his contribution to the project. The authors thank the referees for their valuable comments in improving this paper.

\section{References}

[1] D. Bucco and J. Mazumdar, "Estimation of the fundamental frequencies of shallow shells by a finite element isodeflection contour method", Comput. \& Structures 17 (1983) 441-447.

[2] D. Bucco, J. Mazumdar and G. Sved, "Static analysis of shallow shells of arbitrary shape-A new approach", Internat. J. Numer. Methods Engrg. 18 (1982) 967-979.

[3] L. H. Donnell, Beams, plates and shells (McGraw-Hill, New York, 1976).

[4] P. G. Hodge, The mathematical theory of plasticity (John Wiley \& Sons, New York, 1958).

[5] A. A. Illyushin, Plasticity, (in Russian) (OGIZ, G.I.T.T.L., Moscow, Leningrad, 1948). 
[6] R. K. Jain and J. Mazumdar, "Research note on the elastic plate bending of rectangular plates-A new approach", Int. J. Plasticity 10 (1994) 749-759.

[7] R. Jones and J. Mazumdar, "A method of static analysis of shallow shells", AIAA J. 12 (1974) 1134-1136.

[8] J. Mazumdar, "A method for solving problems of elastic plates of arbitrary shape", J. Aust. Math. Soc. 11 (1970) 95-112.

[9] J. Mazumdar and R. K. Jain, "Elastic plastic analysis of plates of arbitrary shape-A new approach", Int. J. Plasticity 5 (1989) 463-475.

[10] S. Timoshenko and S. Woinowsky-Krieger, Theory of plates and shells (McGraw-Hill, New York, 1959).

[11] V. Z. Vlasov, "General theory of shells and its application in engineering", NASA TT.F-99, Washington, D.C., April, 1964. 